\title{
Prüfung von Verbundfestigkeiten im Mehr-Proben-Versuch
}

—ür die Untersuchung von Klebverbin- dungen, Beschichtungen und Kompositwerkstoffen steht neuerdings eine analytische Zentrifuge zur Verfügung, deren Funktionsweise auf der Idee der Wissenschaftler an der BAM Bundesanstalt für Materialforschung und -prüfung beruht: Bei dieser Methode müssen die Proben nicht mehr eingespannt, sondern nur noch eingesteckt werden, sodass eine sehr schnelle Beladung möglich ist. $\mathrm{Zu}-$ dem können bis zu acht Proben gleichzeitig unter identischen Bedingungen geprüft werden. Konventionelle Zugprüfmaschinen waren und sind nur in der Lage, einen (eingespannten) Probekör-

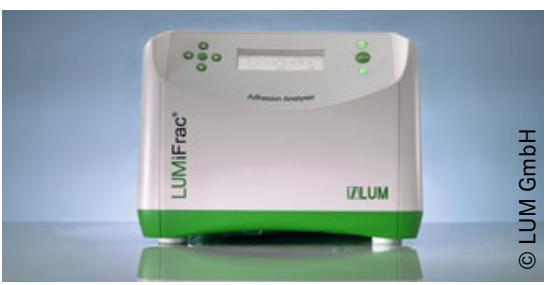

per zu testen. Das von BAM-Forschern bereits 2004 zum Patent angemeldete Funktionsprinzip wurde seitdem von der Berliner LUM GmbH als Lizenznehmer zum marktreifen Produkt weiterentwickelt.

Die resultierende Zeitersparnis liegt im Ergebnis bei etwa 85 Prozent. Wenn die Prüfstempel abreißen beziehungsweise der Verbund versagt, werden der Zeitpunkt, die aktuelle Rotationsgeschwindigkeit und der betroffene Probenplatz automatisch aus der laufenden Zentrifuge an einen Computer gesendet. Eine speziell entwickelte Software berechnet online die kritischen Kräfte und Zugspannungen und stellt die Ergebnisse dar.

Das neue Prüfsystem sieht auf den ersten Blick wie eine herkömmliche Tischzentrifuge aus. In ihr läuft ein von der LUM GmbH entwickelter Spezialrotor mit bis zu 13.000 Umdrehungen pro Minute. Durch aufgeklebte beziehungsweise anderweitig mit dem Prüfkörper verbundene Prüfstempel können Klebungen, Beschichtungen und Kompositwerkstoffe mit Zugkräften belastet werden. Wie bei einem Karussell wird die aus der Drehbewegung resultierende Zentrifugalbeschleunigung ausgenutzt. Erreichbar sind Beschleunigungen von bis zu 10.000 g, wobei Fliehkräfte von bis zu 6,5 kN auf die Proben wirken.

Durch die durchgehend realisierbaren Prüfkräfte von $0,1 \mathrm{~N}$ bis $6,5 \mathrm{kN}$ sind nahezu beliebige Prüfabläufe sowohl für Kurzzeitbeanspruchungs- als auch Langzeitermüdungstests programmierbar.

Der Durchmesser der Prüffläche kann bis zu 10 Millimeter betragen, die maximalen Probenabmessungen liegen derzeit bei $30 \mathrm{~mm}$ x $30 \mathrm{~mm}$ x $15 \mathrm{~mm}$, die dabei prüfbare Zugfestigkeit ist mit bis $\mathrm{zu} 82,5 \mathrm{~N} / \mathrm{mm}^{2}$ angegeben.

Weitere Infos: www.bam.de und www.lum-gmbh.de

\section{Keramikkörper dank 3D-Druck und Klebtechnik}

Ditas urch den Einsatz eines handelsüblichen 3D-Druckers, eines speziellen Keramikpulvers und eines speziellen Klebstoffs ist es der BAM Bundesanstalt für Materialforschung und -prüfung gelungen, ein Knochenersatzgerüst aus Keramik herzustellen. Dazu wird das Kera-

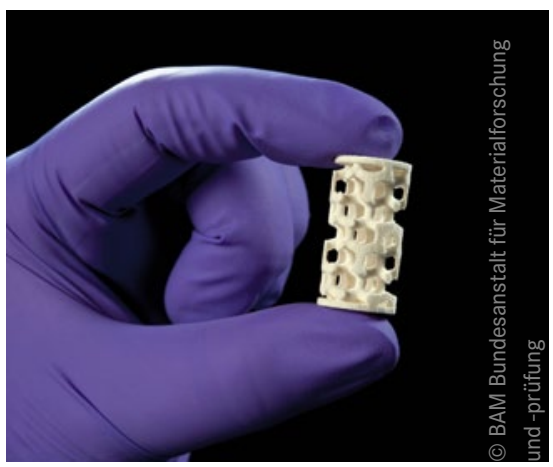

Grünling eines unter Einsatz von Klebstoff mittels 3D-Drucker hergestellten 3D-Gerüsts mikpulver Schicht für Schicht im Drucker aufgetragen, an vorher bestimmten Stellen entsprechend der gewünschten Struktur verklebt und zum Schluss bei circa 1000 Grad Celsius gebrannt.

Der so entstandene Keramikkörper besitzt zwei interessante Eigenschaften: Zum einen besteht er zu 60 Prozent aus Poren, zum anderen kann der Werkstoff vom Körper resorbiert zu werden. Die poröse Struktur ermöglicht den Zellen, in den Körper hineinzuwachsen. Das Material wird dann nach und nach vom Körper abgebaut. Abgeschaut haben die Wissenschaftler die Struktur in der Natur: bei Schwämmen oder bei Hirschgeweihen, die eine ähnliche Knochenstruktur aufweisen.

Die Forscher der BAM verfolgen mit dieser neuen Methode folgendes Ziel: Während einer Operation scannt der Arzt die schadhafte Stelle beim Patien- ten. Nach sofortiger Übertragung der Daten an einen Computer erfolgen die Berechnung des Ersatzteils und der unmittelbare 3D-Druck noch während der OP.

Noch befindet man sich am Anfang der Forschung. Der nächste Schritt ist die Messung der Löslichkeit der Keramikteile, bevor in einer weiteren Phase zusammen mit einem Kooperationspartner Zellzüchtungen durchgeführt werden.

Die Medizintechnik ist aber nur ein Anwendungsfeld der Keramikexperten. Auch in der Automobilindustrie werden zunehmend Keramikmaterialien eingesetzt, um Leichtbau bei gleichzeitig sehr festen Strukturen realisieren zu können. Derzeit wird an einer Norm gearbeitet, die regeln soll, wie viel Festigkeit ein keramisches Bauteil aufweisen muss, um nicht zu versagen.

Weitere Infos: www.bam.de 